\title{
A NUMERICAL MODEL OF THE UNSTEADY FREE BOUNDARY OF AN IDEAL FLUID*
}

\author{
BY \\ PAUL N. SWARZTRAUBER \\ National Center for Almospheric Research, Boulder
}

\begin{abstract}
Unsteady two-dimensional flows which have a free boundary are examined numerically. The fluid is considered to be irrotational and incompressible and the boundary is assumed to have a continuous tangent. The use of numerical techniques enables one to treat the nonlinear problem and to include those cases in which the streamlines intersect the boundary. The technique is quite accurate and calculations are required only on the boundary of the fluid.
\end{abstract}

1. Introduction. Consider a simply connected mass of ideal fluid moving in the plane and subject to a constant pressure on its boundary $B$. Then if at some instant its position and complex velocity potential are known, the problem is to determine its subsequent course.

The problem is formulated in a Lagrangian coordinate system with the solution obtained by following a discrete set of fluid particles located on the surface. The acceleration of the fluid particles is obtained by way of a solution to a singular integral equation which is defined on the boundary. This formulation enables one to restrict all calculations to the boundary of the fluid.

Numerical results are presented for a model problem whose initial boundary is a circle and where the initial velocity potential is $z^{2}$. This problem is also solved by separation of variables with the time-dependent part given as the solution to a nonlinear ordinary differential equation.

In Sec. 4 the two solutions are tabulated in part. A comparison shows a slow error growth rate which starts on the order of $10^{-7}$ and ends on the order of $10^{-6}$, while the boundary undergoes considerable change (Fig. 1).

2. The mathematical formulation. Let $a$ and $b$ denote the Lagrangian coordinates of the fluid at some initial time which we will designate as $t=0$. We will denote the subsequent position of the fluid by the complex function $z(a, b, t)$ and the complex velocity potential by $\psi(a, b, t)$. On the boundary we will also denote the position by $z(s, t)$ where $s$ is a Lagrangian coordinate equal to the arc length along $B$ at $t=0$. The equations governing the motion of the fluid are

$$
(\partial \psi / \partial a)(\partial z / \partial b)-(\partial \psi / \partial b)(\partial z / \partial a)=0
$$

* Received February 1, 1972. The National Center for Atmospheric Research is sponsored by the National Science Foundation. 
for all $a, b, t$

$$
(\overline{\partial z} / \partial t)=(\partial z / \partial a)^{-1}(\partial \psi / \partial a)
$$

and on $B$ for all $s, t$

$$
\operatorname{Re}\left(\overline{\frac{\partial^{2} z}{\partial t^{2}}} \frac{\partial z}{\partial s}\right)=0 .
$$

Since the fluid is assumed to be ideal, $\psi$ must be an analytic function of $z$. Eq. (2.1) is a statement of this fact in the form of the Cauchy-Riemann equations in Lagrangian coordinates.

The derivative $d \psi / d z$ is then independent of the direction in which it is taken and is given by either

$$
d \psi / d z=(\partial z / \partial a)^{-1}(\partial \psi / \partial a)=(\partial z / \partial b)^{-1}(\partial \psi / \partial b)
$$

or, on the boundary,

$$
d \psi / d z=(\partial z / \partial s)^{-1}(\partial \psi / \partial s) .
$$

Eq. (2.2) states that $\psi$ is the velocity potential. The right-hand side of $(2.2)$ is $d \psi / d z$ and hence on the boundary we have

$$
\overline{\partial z} / \partial t=(\partial z / \partial s)^{-1}(\partial \psi / \partial s) .
$$

Eq. (2.3) states that the tangential component of acceleration is zero, which results from the fact that the pressure is constant on the boundary.

The approach will be to determine the acceleration of the boundary, which will then be numerically integrated. Differentiating (2.6), we obtain

$$
\frac{\overline{\partial^{2} z}}{\partial t^{2}}=\left(\frac{\partial z}{\partial s}\right)^{-2}\left(\frac{\partial z}{\partial s} \frac{\partial^{2} \psi}{\partial s \partial t}-\frac{\partial \psi}{\partial s} \frac{\partial^{2} z}{\partial s \partial t}\right) .
$$

If $\partial \psi / \partial t$ were known on the boundary, then the right-hand side could be evaluated and the acceleration could be determined.

It is known that $\partial \psi / \partial t$ can be determined as a solution of Poisson's equation [2]. We will also show that $\partial \psi / \partial t$ can be determined by way of a solution of the Dirichlet problem. This enables us to restrict our attention to the boundary, since the solution of the Dirichlet problem may be obtained by solving an integral equation defined on the boundary.

To this end we define

$$
P \psi=(\partial \psi / \partial t)-(\partial z / \partial t)(\partial z / \partial a)^{-1}(\partial \psi / \partial a) .
$$

$P \psi$ is the derivative of $\psi$ with respect to time at fixed $z . P \psi$ is an analytic function of $z$ which can be proved by showing that it satisfies (2.1) [3]. Now by evaluating (2.8) on the boundary and substituting it into (2.7) we can write the acceleration in terms of the analytic function $P \psi$

$$
\frac{\overline{\partial^{2} z}}{\partial t^{2}}=\left(\frac{\partial z}{\partial s}\right)^{-1}\left(\frac{\partial}{\partial s} P \psi+\frac{\partial z}{\partial t} \frac{\partial}{\partial s} \frac{\overline{\partial z}}{\partial t}\right) \text { on } B .
$$

We shall now proceed to determine $P \psi$. Substituting (2.9) into (2.3) and integrating, we obtain

$$
\operatorname{Re} P \psi=-\frac{1}{2}(\partial z / \partial t)(\overline{\partial z} / \partial t) \text { on } B
$$


It remains to determine the imaginary part of $P \psi$. Let us denote $P \psi=p+i q$ and $z=x+i y$. In the work of Muskhelishvili [1] it is shown that $P \psi$ is analytic on $D$ and continuous on $D \cup B$ if and only if $p$ and $q$ satisfy the integral equation

$$
q(z)=\frac{1}{\pi} \int_{B} q(\zeta) \operatorname{Im} \frac{d \zeta}{\zeta-z}-\frac{1}{\pi} P V \int_{B} p(\zeta) \operatorname{Re} \frac{d \zeta}{\zeta-z}
$$

where $\zeta, z \in B$. Now given $p$ we can determine $q$ from (2.11). Then $P \psi=p+i q$ substituted into (2.9) gives the acceleration. The numerical solution of (2.11) is discussed in detail in the paper by Swarztrauber [4] and will be discussed briefly in Sec. 3.

We note that since $\mathrm{P} \psi$ is analytic, $P^{2} \psi$ is also analytic, and in general $P^{n} \psi$ is analytic. In theory then, if the initial data are sufficiently differentiable, all the time derivatives of $z$ could be determined from a sequence of solutions of Dirichlet problems. This leads to a series representation of $z$, and the existence of a local solution could be established by proving convergence of this series. If the initial data are not analytic, then for some $n, P^{n} \psi$ could have a singularity on the boundary and rupture would be likely for $t>0$. The construction of the series for $z$ is not practical, since, in general, closed-form solutions to the Dirichlet problem do not exist and the amount of differentiation is prohibitive. Hence, we turn to numerical methods.

3. The numerical formulation. Let $z\left(s_{i}, 0\right)=z\left(a_{i}, b_{i}, 0\right)=a_{i}+i b_{i}$ be a set of points which approximate the boundary at $t=0$. Then the numerical solution is obobtained by determining values $z_{j}^{n}$ and $\partial z^{n} / \partial t_{j}$ which approximate $z\left(s_{i}, n \Delta t\right)$ and $(\partial z / \partial t)\left(s_{i}, n \Delta t\right)$. The complex velocity potential $\psi(a, b, t)$ is not carried in the computation; however, it can be determined by integrating $\partial z / \partial t=d \psi / d z$ with respect to $z$.

We can now state the sequence of computations. Subscripts are omitted; however, it is assumed that the equations are evaluated at a discrete set of points corresponding to $s_{i}, j=1,2, \cdots, L$, and at an arbitrary time level $n$. The details of each step in the sequence will follow.

1. Compute $p=\operatorname{Re} P \psi=-\frac{1}{2}(\partial z / \partial t)(\overline{\partial z} / \partial t)$.

2. Compute $q=\operatorname{Im} P \psi$ as a solution to (2.11).

3. Compute the acceleration of the boundary $\partial^{2} z / \partial t^{2}$ from (2.9).

4. Numerically integrate $\partial^{2} z / \partial t^{2}$ in time and return to step 1 .

For $t=0$ the right side of the equation in step 1 is computed from the initial conditions, and for $t>0$ it is computed from the integrated values of the velocity.

In step 2 we obtain $q=\operatorname{Im} P \psi$ by Picard iteration. Starting from an arbitrary $q_{0}(z)$, we define

$$
q_{m+1}(z)=\frac{1}{\pi} \int_{B} q_{m}(z) \operatorname{Im} \frac{d \zeta}{\zeta-z}-\frac{1}{\pi} \int_{B}[p(\zeta)-p(z)] \operatorname{Re} \frac{d \zeta}{\zeta-z} .
$$

In practice this iteration converges rapidly to the solution $q(z)$. By using $q(z)$ at previous time levels one can obtain a good initial guess $q_{0}(z)$, after which only two or three iterations are required for the experiment in Sec. 4.

For computational purposes it is convenient to express (3.1) in terms of real functions. If we denote $\zeta$ as $x(s)+i y(s)$ and use dot notation to refer to differentiation with respect to $s$, then

$[p(\zeta)-p(z)] \operatorname{Re} \frac{d \zeta}{\zeta-z}=[p(s)-p(r)] \frac{[x(s)-x(r)] \dot{x}(s)+[y(s)-y(r)] \dot{y}(s)}{[x(s)-x(r)]^{2}+[y(s)-y(r)]^{2}} d s$, 
and at $\zeta=z$

$$
\begin{gathered}
{[p(\zeta)-p(z)] \operatorname{Re} \frac{d \zeta}{\zeta-z}=\dot{p}(r) d s} \\
q_{m}(s) \operatorname{Im} \frac{d \zeta}{\zeta-z}=q_{m}(s) \frac{[x(s)-x(r)] \dot{y}(s)-[y(s)-y(r)] \dot{x}(s)}{[x(s)-x(r)]^{2}+[y(s)-y(r)]^{2}} d s
\end{gathered}
$$

and at $\zeta=z$

$$
q_{m}(\zeta) \operatorname{Im} \frac{d \zeta}{\zeta-z}=\frac{q_{m}(r)}{2} \frac{\dot{x}(r) \ddot{y}(r)-\dot{y}(r) \ddot{x}(r)}{\dot{x}(r)^{2}+\dot{y}(r)^{2}} .
$$

In Eqs. (3.2) through (3.5), as well as in (2.9), it is required to evaluate numerically derivatives with respect to $s$. This calculation, together with that of numerical quadrature, can be simplified by changing the definition of $s$. This is possible since the equations are independent of this definition. Therefore, instead of $s$ being arc length along $B$ at $t=0$, we will select $s$ as the point number. That is, $s$ will be a continuous real function defined on $B$ which assumes integer values at the points where the boundary is tabulated; specifically, at $s_{j}=s\left(z_{i}\right)=j$. The functions are then tabulated at equal intervals $\Delta s=1$. The derivative with respect to point numbers may be approximated by the following five-point formulas:

$$
\begin{aligned}
& \dot{x}\left(s_{i}\right) \simeq \frac{1}{12}\left[S\left(x_{i+1}-x_{i-1}\right)+x_{j-2}-x_{j+2}\right], \\
& \ddot{x}\left(s_{j}\right) \simeq \frac{1}{12}\left[16\left(x_{i+1}+x_{i-1}\right)-x_{i+2}-x_{i-2}-30 x_{i}\right] .
\end{aligned}
$$

These formulas are used in Sec. 4 and give six decimal digits of accuracy. Four other numerical differentiation methods were evaluated. A three-point difference formula gave only three decimal digits of accuracy. However, the same margin was not gained by going from the five-point to a seven-point formula. A periodic cubic spline was comparable in accuracy to the five-point formula. This approximation was possible since all the functions defined on $B$ are periodic in $s$. Finally, it was hoped that a Fourier series representation via the fast Fourier transformation would provide the best results; however, it produced unstable calculations. This may in part be explained by the fact that a formal differentiation of a Fourier series will amplify the high frequencies relative to the series obtained by applying a finite-difference approximation to the derivative.

Now, by using (3.6) and (3.7), the integrands may be tabulated at $s_{j}=j$, and the trapezoidal rule used to evaluate the integrals in (3.1). For sufficiently differentiable periodic functions, the trapezoidal rule is the best possible in the sense that it is as accurate as any other numerical quadrature which is exact for the constant function. The function $q=\operatorname{Im} P \psi$ can now be determined from (3.1) which completes step 2 .

In step 3 the acceleration of the boundary is determined. If we denote the velocity as $\partial z / \partial t=u(s)+i v(s)$, then Eq. (2.9) becomes

$$
\frac{\partial^{2} x}{\partial t^{2}}-i \frac{\partial^{2} y}{\partial t^{2}}=\frac{\dot{p}(s)+i \dot{q}(s)+[u(s)+i v(s)][\dot{u}(s)-i \dot{v}(s)]}{\dot{x}(s)+i \dot{y}(s)}
$$

which completes step 3. 
Step 4 consists of the numerical integration of the acceleration to obtain the pusition and velocity of the boundary for $t>0$. The following two-level implicit scheme was selected on the basis of its desirable stability characteristics and relatively high degree of accuracy.

Predictor:

$$
\begin{gathered}
\partial z^{n+1} / \partial t=\left(\partial z^{n-1} / \partial t\right)+2 \Delta t\left(\partial^{2} z^{n} / \partial t^{2}\right) \\
z^{n+1}=z^{n-1}+2 \Delta t \frac{\partial z^{n-1}}{\partial t}+\frac{2}{3} \Delta t^{2}\left(\frac{\partial^{2} z^{n-1}}{\partial t^{2}}+2 \frac{\partial^{2} z^{n}}{\partial t^{2}}\right)
\end{gathered}
$$

Corrector:

$$
\begin{gathered}
\partial z^{n+1} / \partial t=\left(\partial z^{n} / \partial t\right)+\frac{1}{2} \Delta t\left(\left(\partial^{2} z^{n} / \partial t^{2}\right)+\left(\partial^{2} z^{n+1} / \partial t^{2}\right)\right) \\
z^{n+1}=z^{n}+\Delta t\left(\partial z^{n} / \partial t\right)+\frac{1}{6} \Delta t^{2}\left(2\left(\partial^{2} z^{n} / \partial t^{2}\right)+\left(\partial^{2} z^{n+1} / \partial t^{2}\right)\right)
\end{gathered}
$$

The scheme is made implicit at the expense of iterating (3.11) and (3.12) until convergence. However, in practice, if $\Delta t$ was chosen to provide the desired accuracy, then on the average only two or three iterations were required.

4. A numerical experiment. The initial boundary of the fluid is a circle of unit radius which is described by 128 points.

$$
\begin{aligned}
& x(s)=\cos ([2 \pi / 128] s), \quad s=0,1,2, \cdots, 127, \\
& y(s)=\sin ([2 \pi / 128] s), \quad s=0,1,2, \cdots, 127 .
\end{aligned}
$$

The initial velocity potential of the fluid is selected to be $\psi=z^{2}$. Hence on the boundary

$$
\begin{aligned}
& u(s)=2 \cos ([2 \pi / 128] s), \quad s=0,1,2, \cdots, 127, \\
& v(s)=-2 \sin ([2 \pi / 128] s), \quad s=0,1,2, \cdots, 127
\end{aligned}
$$

The evolution of the fluid is shown in Fig. 1. The quantitative results are given in Table 1 for several values of $s$ corresponding to points initially located at angles of

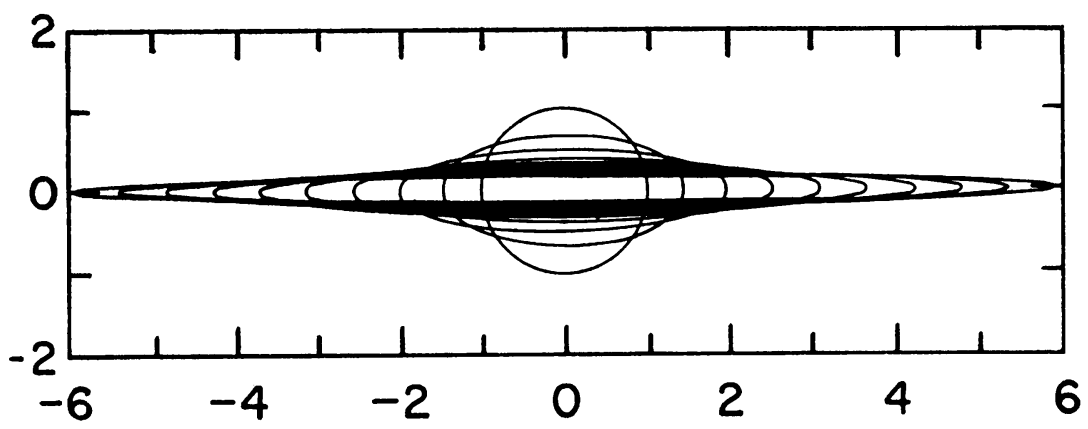

Fig. $1 . \quad B(t)$ for $t=0, .2, .4, \cdots, 1.8$. 
TABLE 1

\begin{tabular}{ccccc}
\hline$t$ & $x(1)$ & $x(17)$ & $y(17)$ & $y(33)$ \\
0.0 & 1.00000000 & 0.70710678 & 0.70710678 & 1.00000000 \\
0.2 & 1.46477385 & 1.03575149 & 0.48274119 & 0.68269901 \\
0.4 & 1.99930229 & 1.41372009 & 0.35367682 & 0.50017382 \\
0.6 & 2.55424647 & 1.80612481 & 0.27683603 & 0.39150370 \\
0.8 & 3.11546126 & 2.20296352 & 0.22696737 & 0.32097797 \\
1.0 & 3.67898674 & 2.60143614 & 0.19220199 & 0.27181153 \\
1.2 & 4.24350779 & 3.00061273 & 0.16663314 & 0.23565096 \\
1.4 & 4.80851195 & 3.40013093 & 0.14705369 & 0.20796070 \\
1.6 & 5.37377282 & 3.79983068 & 0.13158534 & 0.18608449 \\
1.8 & 5.93917999 & 4.19963400 & 0.11905843 & 0.16836856 \\
\hline
\end{tabular}

$0^{\circ}, 45^{\circ}$, and $90^{\circ}$. This table was prepared using an interval of integration $\Delta t=.001$ and required 4 minutes of computing time on the Control Data 7600. For $t>1.8$ the boundary is quite distorted and the error growth rate increases.

This problem may also be solved in an alternate manner which provides a useful comparison. Assume a velocity potential of the form $\psi=\frac{1}{4}(d f / d t) z^{2}$ where $f$ is a function of $t$ only and is yet to be determined. Then by integrating (2.6) with respect to time, we obtain

$$
\begin{aligned}
& x(s, t)=e^{1 / 2 f} \cos ([2 \pi / 128] s), \\
& y(s, t)=e^{-1 / 2 f} \sin ([2 \pi / 128] s),
\end{aligned}
$$

which gives

$$
\begin{aligned}
& u(s, t)=\frac{1}{2}(d f / d t) e^{1 / 2 f} \cos ([2 \pi / 128] s), \\
& v(s, t)=-\frac{1}{2}(d f / d t) e^{-1 / 2 f} \sin ([2 \pi / 128] s) .
\end{aligned}
$$

Now $P \psi=\frac{1}{4}\left(d^{2} f / d t^{2}\right) z^{2}$, which yields

$$
p(s, t)=\frac{1}{4} \frac{d^{2} f}{d t^{2}}\left[e^{s} \cos ^{2}\left(\frac{2 \pi}{128} s\right)-e^{-s} \sin ^{2}\left(\frac{2 \pi}{128} s\right)\right] .
$$

Using (3.8), we find that (2.3) is satisfied if

$$
\left(d^{2} f / d t^{2}\right)+\frac{1}{2}(d f / d t) \tanh f=0
$$

with the initial conditions $f(0)=0$ and $(d f / d t)(0)=4$, which are obtained from (4.5) and (4.7).

Eq. (4.10) may be integrated once to yield

$$
d f / d t=4(\cosh f)^{-1 / 2}
$$

which may now be numerically integrated to yield $f$. Substituting $f$ into (4.5) and (4.6), we obtain Table 2 for comparison with Table 1. 
TABle 2

\begin{tabular}{ccccc}
\hline$t$ & $x(1)$ & $x(17)$ & $y(17)$ & $y(33)$ \\
0.0 & 1.00000000 & 0.70710678 & 0.70710678 & 1.00000000 \\
0.2 & 1.46477382 & 1.03575150 & 0.48274128 & 0.68269926 \\
0.4 & 1.99930195 & 1.41371997 & 0.35367683 & 0.50017457 \\
0.6 & 2.55424577 & 1.80612450 & 0.27683584 & 0.39150501 \\
0.8 & 3.11546019 & 2.20296303 & 0.22696704 & 0.32097986 \\
1.0 & 3.67898532 & 2.60143547 & 0.19220158 & 0.27181408 \\
1.2 & 4.24350601 & 3.00061187 & 0.16663268 & 0.23565420 \\
1.4 & 4.80850981 & 3.40012990 & 0.14705321 & 0.20796464 \\
1.6 & 5.37377036 & 3.79982946 & 0.13158485 & 0.18608908 \\
1.8 & 5.93917740 & 4.19963261 & 0.11905803 & 0.16837349 \\
\hline
\end{tabular}

I would like to express my sincere appreciation to Professor Robert D. Richtmyer of the University of Colorado. This paper contains a portion of my dissertation which was written under his chairmanship.

\section{REFERENCES}

[1] N. I. Muskhelishvili, Singular integral equations, P. Noordhoff, Gröningen, Holland, p. 61 (1953)

[2] F. V. Pohle, Motion of water due to breaking of a dam and related problems, in Proc. National Bureau of Standards Semicentennial Symposium on Gravity Waves, NBS Circular 521, Washington, D. C., pp. 47-53 (1952)

[3] P. N. Swarztrauber, $A$ study of the time-dependent free boundary of an ideal fluid, Ph.D. thesis, University of Colorado, Boulder, Colorado, 1970

[4] P. N. Swarztrauber, On the numerical solution of the Dirichlet problem for a region of general shape, SIAM J. Numer. Anal. 9, 300-306 (1972) 